\title{
Atividades físicas no lazer entre adultos de Florianópolis, Santa Catarina, Brasil: estudo populacional sobre as características das práticas e de seus praticantes
}

\author{
Leisure-time physical activities among adults in Florianopolis, \\ state of Santa Catarina, Brazil: a population-based study \\ on the characteristics of the practices and the practitioners
}

Giovâni Firpo Del Duca ${ }^{1}$

Markus Vinicius Nahas ${ }^{1}$

Pedro Curi Hallal ${ }^{2}$

Karen Glazer Peres ${ }^{3}$
${ }^{1}$ Centro de Desportos, Universidade Federal de Santa Catarina. Campus Universitário Centro de Desportos Coordenadoria de Pós-Graduação em Educação Física, Trindade. 88040-900 Florianópolis SC Brasil.

gfdelduca@gmail.com

${ }^{2}$ Programa de Pós-

Graduação em

Epidemiologia,

Universidade Federal de

Pelotas.

${ }^{3}$ University of Adelaide.

\begin{abstract}
The study established characteristics of practices and practitioners of leisure-time physical activities among adults in Florianopolis, Brazil. It was a population-based, cross-sectional survey conducted between September 2009 and January 2010. Leisure-time physical activity was assessed using a validated questionnaire. Walking and weight lifting were the most frequently reported activities. Vigorous physical activities were more frequent among men and young adults. There were no gender or age differences in relation to the frequency and duration of physical activities. Walking and gymnastics were more frequently practiced by women, and jogging and soccer by men. Age was positively associated with walking, and negatively with soccer and weight lifting. Caucasian and black males practiced gymnastics and soccer more frequently. Adults living with a partner practiced more walking and soccer, and less weight lifting. Educational level was directly associated with jogging and gymnastics, but inversely with soccer. Income was inversely associated with soccer and directly associated with weight lifting. Policies to promote physical activity should consider the different interests of the population to stimulate the scope of their practice in leisure-time.
\end{abstract}

Key words Leisure activities, Motor activity, Exercise, Sports, Epidemiological studies, Cross-sectional studies
Resumo O presente estudo determinou as características das práticas e dos praticantes de atividades físicas no lazer entre adultos de Florianópolis, Santa Catarina. Inquérito transversal de base populacional de setembro/2009 a janeiro/2010. A atividade fisica no lazer foi determinada por meio de questionário validado. Caminhada e musculação foram as atividades mais relatadas. Homens e adultos mais jovens praticaram mais atividades físicas vigorosas. Não foram observadas diferenças de gênero ou idade em relação à frequência e à duração das atividades físicas. Caminhada e ginástica foram mais praticadas por mulheres. Corrida e futebol foram mais praticados por homens. A idade associou-se diretamente ao relato de caminhada e, inversamente, ao futebol e musculação. Brancos e negros apresentaram, respectivamente, maiores práticas de ginástica e futebol. Adultos com companheiro(a) praticaram mais caminhada e futebol, e menos musculação. A escolaridade associou-se diretamente com práticas de corrida e ginástica, e inversamente com o futebol. A renda associou-se inversamente com o relato do futebol, e diretamente com a prática de musculação. Ações de promoção da atividade física devem considerar os distintos interesses populacionais para o estímulo à sua prática no lazer.

Palavras-chave Atividades de lazer, Atividade motora, Exercício, Esportes, Estudos epidemiológicos, Estudos transversais 


\section{Introdução}

Os efeitos deletérios da inatividade física repercutem globalmente na ocorrência de 6-10\% das doenças crônicas não transmissíveis ${ }^{1}$ e a colocam no patamar de segundo lugar dentre todos os comportamentos de risco responsáveis pela mortalidade populacional, ficando atrás apenas do tabagismo². Embora nas últimas décadas os níveis de inatividade física tenham se elevado consideravelmente ${ }^{3}$, a investigação dos seus diferentes domínios tem apontado para algumas especificidades, como a tendência de redução desse comportamento de risco no âmbito do lazer ${ }^{4,5}$.

Dentre todos os domínios da atividade física (deslocamento, domicílio, lazer e trabalho), aquela praticada, o lazer colabora para as maiores reduções do risco de mortalidade por todas as causas e por doenças cardiovasculares ${ }^{6}$. Essa constatação respalda a importância de tradicionais sistemas de monitoramento, como o Behavioral Risk Factor Surveillance System ${ }^{7}$, nos Estados Unidos, e o Sistema de Vigilância de Fatores de Risco e Proteção para Doenças Crônicas por Inquérito Telefônico ${ }^{8}$, no Brasil, adotarem esse indicador para o monitoramento das condições de saúde de suas populações.

Estudos nacionais no campo da epidemiologia da atividade física dispostos a investigar o domínio do lazer têm apontado que aproximadamente um quarto da população adulta é regularmente ativa ${ }^{9} \mathrm{e}$, quando o ponto de corte preconiza o alcance das recomendações semanais de atividades físicas exclusivamente nesse domínio, essa proporção é reduzida consideravelmente, sofrendo interferência de diversos aspectos sociodemográficos ${ }^{8}$. Muito embora o lazer seja o domínio da atividade física mais explorado na literatura, as pesquisas desenvolvidas comumente centram-se em sua prática generalizada, sem explorar outros aspectos igualmente relevantes como a duração, a frequência, a intensidade, os principais tipos de prática e o perfil de seus praticantes. Conhecer e reconhecer tais atividades físicas e as diferenças demográficas e sociais de seus praticantes certamente auxiliará na elaboração de políticas públicas voltadas à promoção da atividade física nesse domínio. O objetivo deste estudo foi determinar a duração, a frequência, a intensidade, o tipo e o perfil sociodemográfico dos adultos praticantes das principais atividades físicas realizadas no lazer em Florianópolis, Santa Catarina.

\section{Métodos}

O presente estudo teve delineamento transversal de base populacional e está vinculado ao inquérito EpiFloripa, desenvolvido com adultos de 20 a 59 anos em Florianópolis, Santa Catarina. O município destaca-se por apresentar o terceiro melhor índice de desenvolvimento humano do país, assumindo a primeira posição dentre todas as capitais brasileiras avaliadas ${ }^{10}$.

O cálculo de tamanho amostral considerou os seguintes parâmetros e estimativas: prevalência de diferentes desfechos do inquérito de $50 \%$, nível de confiança de $95 \%$, erro aceitável de quatro pontos percentuais, efeito de delineamento amostral igual a dois e adicional de $10 \%$ por motivo de perdas. Ao valor obtido, foram acrescidos $15 \%$ para o controle de confusão em análises de associação ajustadas. Após arredondamento, a amostra requerida foi de 2016 adultos.

O processo de amostragem ocorreu em dois estágios. As unidades amostrais primárias foram os setores censitários da zona urbana do município, definidos pelo Instituto Brasileiro de Geografia e Estatística ${ }^{11}$. De um total de 420 setores existentes, ordenados de forma crescente à renda média dos chefes dos domicílios, sorteou-se sistematicamente 60 deles. Em seguida, o número de domicílios em cada um dos setores selecionados foi atualizado, devido à inexistência de dados atualizados. De posse da listagem dos domicílios ocupados, foram selecionados sistematicamente 18 unidades em cada setor censitário. Todos os moradores dos domicílios sorteados com idade de 20 a 59 anos de idade foram inicialmente incluídos. Foram excluídos os adultos institucionalizados ou inaptos a responder ao questionário por conta própria no momento da entrevista.

A atividade física no lazer foi avaliada pelo questionário do Sistema de Vigilância de fatores de risco e proteção para doenças crônicas por inquérito telefônico - Vigitel - do ano $2009^{8}$. O principal tipo de atividade física praticada no lazer foi investigado por duas questões: "Nos últimos três meses, o(a) sr(a) praticou algum tipo de exercício físico ou esporte?" e "Qual o tipo principal de exercício físico ou esporte que o(a) sr(a) praticou?".

A prática regular de atividade física nesse domínio foi definida pela resposta positiva à questão: " $\mathrm{O}(\mathrm{A})$ Sr.(a) pratica o exercício ou esporte pelo menos uma vez por semana?". Todos os adultos regularmente ativos no lazer foram indagados quanto à duração e frequência da principal atividade física relatada. Para isso, foram realizados, 
respectivamente, os seguintes questionamentos: "Quantos dias por semana o(a) sr(a) costuma praticar exercício físico ou esporte?" e "No dia que o(a) sr(a) pratica exercício ou esporte, quanto tempo dura esta atividade?". Com relação à intensidade, foram classificadas como práticas leves ou moderadas a caminhada, caminhada em esteira, musculação, hidroginástica, ginástica em geral, natação, artes marciais, ciclismo e voleibol. Já as atividades definidas como práticas de intensidade vigorosa foram: corrida, corrida em esteira, ginástica aeróbica, futebol e basquetebol ${ }^{8}$. Outras respostas relatadas apresentaram um baixo percentual de respostas e, por não terem sido previamente discriminadas no questionário, não foram incluídas na análise relacionada à intensidade das atividades físicas.

As variáveis sociodemográficas avaliadas foram: gênero, idade (em anos), situação conjugal (com companheiro ou sem companheiro), escolaridade (em anos completos de estudo) e renda familiar per capita (em quartil).

Os dados foram coletados no período de setembro/2009 a janeiro/2010 por 36 entrevistadoras devidamente treinadas. Empregou-se o Personal Digital Assistant (PDA) modelo Mio P550b (Mio Technology Corporation, Estados Unidos) para a aplicação de entrevistas face a face. Realizou-se um controle de qualidade em 15\% dos questionários concluídos, a partir da aplicação de uma versão reduzida do instrumento via telefone na semana seguinte.

A análise estatística foi realizada no Stata - versão 11.0 (Stata Corporation, College Station, Estados Unidos). Os pesos amostrais do estudo foram atribuídos a partir do inverso da probabilidade do domicílio ser sorteado no setor censitário ao qual pertencia. Considerou-se a amostra complexa pelo emprego do comando "svy". A estatística descritiva incluiu estimativas de prevalências e intervalos de confiança de 95\% (IC95\%). A duração das atividades físicas praticadas no lazer conforme o gênero e a idade foi testada pelo emprego da regressão multinomial. A intensidade e a frequência das práticas conforme o gênero e a idade foram testadas pelo uso da regressão de Poisson. Os valores $\mathrm{p}$ foram resultantes de análises ajustadas, controlando-se cada associação para as demais variáveis dependentes e independentes supracitadas.

Na determinação dos fatores sociodemográficos associados aos cinco principais tipos de atividades físicas praticadas no lazer, foi empregada a regressão de Poisson nas análises bruta e ajustada. Os ajustes no modelo de análise incluíram as variáveis demográficas (gênero, idade, situação conjugal atual e cor da pele) em nível distal e as variáveis sociais (escolaridade e renda familiar per capita) em nível proximal. Na modelagem estatística foi adotada a estratégia de seleção para trás e um nível crítico de $\mathrm{p} \leq 0,20$ para permanência no modelo, com intuito de controle de potenciais fatores de confusão. O efeito de cada indicador sociodemográfico sobre os desfechos foi ajustado para as demais variáveis do mesmo nível ou de nível superior.

O estudo foi aprovado pelo Comitê de Ética em Pesquisa com Seres Humanos da Universidade Federal de Santa Catarina, Brasil.

\section{Resultados}

Foram entrevistados 1.720 adultos, sendo 761 homens e 959 mulheres. Considerando o número de sujeitos elegíveis $(n=2.016)$, o estudo apresentou um percentual de respostas de 85,3\%. A Tabela 1 apresenta as características sociodemográficas e a prática regular de atividade física no lazer, conforme gênero. A faixa etária mais comum observada em ambos os gêneros foi de 20 a 29 anos $(35,5 \%$ dos homens e $31,8 \%$ das mulheres). As características sociodemográficas foram muito semelhantes entre os gêneros, com exceção da renda familiar per capita, mais elevada entre os homens $(\mathrm{p}=0,002)$. A prática regular de atividade física no lazer foi significativamente maior entre os homens (54,5\% versus $41,9 \%$ das mulheres; $\mathrm{p}<0,001)$.

Os principais tipos de atividades físicas praticadas pelos adultos de Florianópolis são apresentados na Tabela 2. Um total de 885 adultos $(52,2 \%)$ relatou ter praticado algum tipo de atividade física no lazer nos últimos três meses (59,7\% dos homens e 46,2\% das mulheres). $\mathrm{Na}$ totalidade da amostra, o principal tipo de atividade física realizada foi a caminhada $(32,9 \%)$, seguida pela musculação $(16,7 \%)$ e pelo futebol $(15,3 \%)$. Entre os homens, o futebol $(28,5 \%)$, a caminhada $(21,8 \%)$ e a musculação $(16,8 \%)$ foram as atividades físicas mais relatadas e, entre as mulheres, a caminhada $(44,5 \%)$ foi seguida pela musculação $(16,6 \%)$ e ginástica $(11,6 \%)$.

As características das atividades físicas praticadas no lazer conforme o gênero são apresentadas na Figura 1. Os homens relataram práticas de intensidade vigorosa em maior proporção que as mulheres (42,7\% e 13,6\%, respectivamente; $\mathrm{p}$ $<0,001)$, que relataram mais frequentemente as atividades físicas leves e moderadas $(86,4 \%$ ver- 
Tabela 1. Características sociodemográficas e atividade física no lazer conforme gênero em adultos. Florianópolis, Santa Catarina, 2010.

\begin{tabular}{|c|c|c|c|c|c|}
\hline \multirow{2}{*}{ Variável } & \multicolumn{2}{|c|}{ Homens $(n=761)$} & \multicolumn{2}{|c|}{ Mulheres $(n=959)$} & \multirow[b]{2}{*}{ Valor $\mathbf{p}$} \\
\hline & $\mathbf{n}$ & $\%^{\mathrm{a}}$ & $\mathbf{n}$ & $\%^{a}$ & \\
\hline Idade (anos completos) & & & & & 0,13 \\
\hline $20-29$ & 260 & 35,5 & 280 & 31,8 & \\
\hline $30-39$ & 172 & 23,1 & 220 & 23,6 & \\
\hline $40-49$ & 181 & 23,9 & 257 & 26,3 & \\
\hline$\geq 50$ & 148 & 17,5 & 202 & 18,3 & \\
\hline Cor da pele autorreferida ${ }^{\mathrm{b}}$ & & & & & 0,28 \\
\hline Branca & 642 & 85,2 & 802 & 86,3 & \\
\hline Parda & 74 & 10,3 & 73 & 8,2 & \\
\hline Preta & 34 & 4,5 & 53 & 5,6 & \\
\hline Situação conjugal atual & & & & & 0,77 \\
\hline Com companheiro(a) & 302 & 39,8 & 375 & 40,6 & \\
\hline Sem companheiro(a) & 459 & 60,2 & 584 & 59,4 & \\
\hline Escolaridade (anos completos) & & & & & 0,54 \\
\hline$\leq 4$ & 71 & 8,2 & 90 & 7,8 & \\
\hline 5 a 8 & 108 & 13,4 & 145 & 13,8 & \\
\hline 9 a 11 & 263 & 34,8 & 305 & 32,9 & \\
\hline$\geq 12$ & 318 & 43,6 & 419 & 45,5 & \\
\hline Renda familiar per capita (quartis) ${ }^{c}$ & & & & & 0,002 \\
\hline $1^{\circ}$ (menor) & 203 & 26,7 & 305 & 30,9 & \\
\hline $2^{\circ}$ & 152 & 18,6 & 183 & 19,4 & \\
\hline $3^{\circ}$ & 193 & 27,2 & 228 & 25,0 & \\
\hline $4^{\circ}$ (maior) & 197 & 27,5 & 224 & 24,7 & \\
\hline Prática regular de atividade física no lazer & & & & & $<0,001$ \\
\hline Não & 354 & 45,5 & 558 & 58,1 & \\
\hline Sim & 406 & 54,5 & 400 & 41,9 & \\
\hline
\end{tabular}

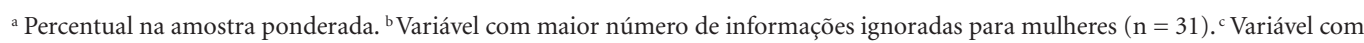
maior número de informações ignoradas para homens $(\mathrm{n}=16)$.

Tabela 2. Principais tipos de atividade física praticadas no lazer por adultos, conforme gênero. Florianópolis, Santa Catarina, 2010.

\begin{tabular}{|c|c|c|c|c|c|c|}
\hline \multirow{2}{*}{ Tipo de atividade física } & \multicolumn{2}{|c|}{ Total $(\mathbf{n}=\mathbf{8 8 5})$} & \multicolumn{2}{|c|}{ Homens $(n=448)$} & \multicolumn{2}{|c|}{ Mulheres $(n=437$} \\
\hline & $\mathbf{n}$ & $\%^{\mathrm{a}}$ & $\mathbf{n}$ & $\%^{\mathrm{a}}$ & $\mathbf{n}$ & $\%^{\mathrm{a}}$ \\
\hline Basquete & 1 & 0,1 & 1 & 0,2 & - & - \\
\hline Caminhada & 311 & 32,9 & 104 & 21,8 & 207 & 44,5 \\
\hline Ciclismo & 34 & 3,9 & 23 & 5,2 & 11 & 2,5 \\
\hline Corrida & 59 & 6,5 & 42 & 8,6 & 17 & 4,2 \\
\hline Futebol & 135 & 15,3 & 129 & 28,5 & 6 & 1,5 \\
\hline Ginástica & 57 & 6,8 & 8 & 2,2 & 49 & 11,6 \\
\hline Hidroginástica & 14 & 1,3 & 2 & 0,4 & 12 & 2,3 \\
\hline Lutas & 13 & 1,5 & 8 & 1,9 & 5 & 1,2 \\
\hline Musculação & 141 & 16,7 & 73 & 16,8 & 68 & 16,6 \\
\hline Natação & 27 & 3,3 & 17 & 4,1 & 10 & 2,5 \\
\hline Tênis & 10 & 1,3 & 9 & 2,3 & 1 & 0,2 \\
\hline Vôlei & 5 & 0,8 & 1 & 0,3 & 4 & 1,2 \\
\hline Outros & 78 & 9,6 & 31 & 7,6 & 47 & 11,6 \\
\hline
\end{tabular}

a Percentual na amostra ponderada. 
sus $57,3 \%$ dos homens). Não foram observadas diferenças estatisticamente significativas entre os gêneros quanto à duração e frequência semanal das atividades físicas de lazer.

Os adultos mais jovens, com 20 a 39 anos de idade, também relataram mais frequentemente as atividades físicas de maior intensidade $(33,2 \%)$ quando comparados àqueles de 40 a 59 anos $(21,3 \%, p=0,04)$. A duração e a frequência semanal das atividades físicas no lazer foram semelhantes conforme as diferentes faixas etárias (Figura 2).
A caracterização dos praticantes dos principais tipos de atividade física relatadas no lazer é apresentada na Tabela 3. A prática de caminhada e ginástica foi mais frequente entre as mulheres, enquanto a corrida e o futebol foram observados em maior proporção entre os homens. Adultos mais jovens apresentaram uma maior probabilidade de praticar futebol e musculação, assim como uma menor probabilidade de praticar caminhada quando comparados àqueles mais velhos. Com relação à cor da pele, sujeitos que relataram ter cor da pele parda e negra pratica-

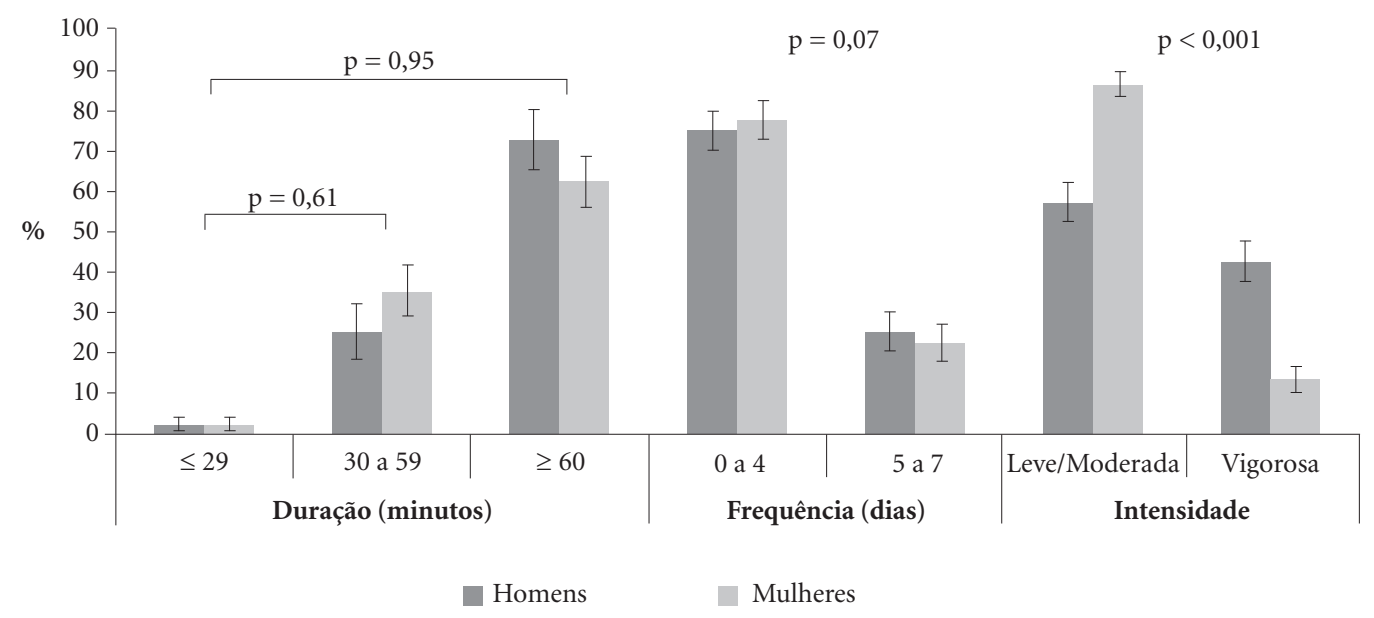

Figura 1. Características das atividades físicas no lazer conforme gênero em adultos de Florianópolis, Santa Catarina, 2010.

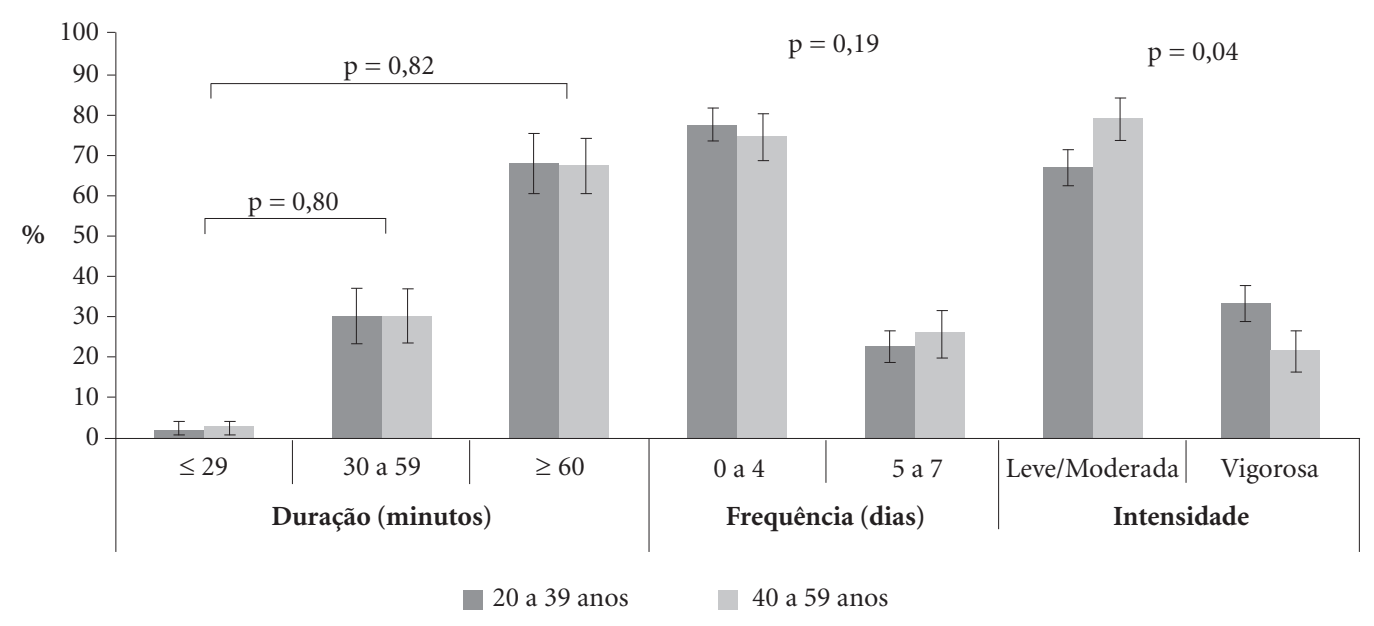

Figura 2. Características das atividades físicas no lazer conforme idade em adultos de Florianópolis, Santa Catarina, 2010. 
Tabela 3. Caracterização dos adultos praticantes dos principais tipos de atividades físicas de lazer. Florianópolis, Santa Catarina, 2010.

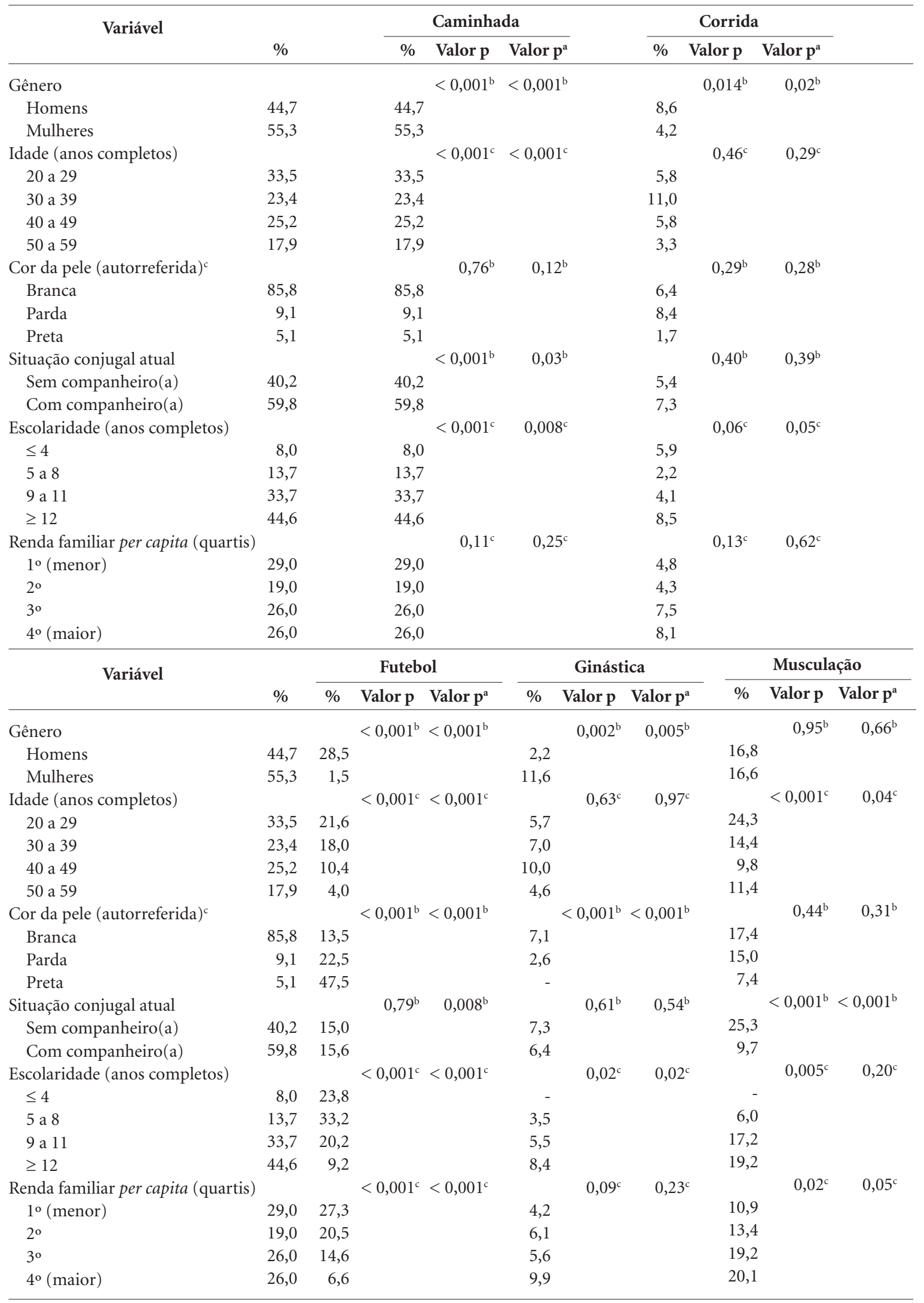

a Valor p resultante de análise ajustada para gênero, idade, cor da pele e situação conjugal (primeiro nível); escolaridade e renda familiar per capita (segundo nível). ${ }^{\mathrm{b}}$ Valor $\mathrm{p}$ resultante de teste de heterogeneidade. ${ }^{\mathrm{c}}$ Valor $\mathrm{p}$ resultante de teste de tendência linear. 
vam o futebol com maior frequência e a ginástica com menor frequência em relação àqueles com cor da pele branca. Adultos com companheiro(a) apresentaram maior probabilidade de praticar a caminhada e o futebol e menor probabilidade de praticar musculação quando confrontados com aqueles que vivem sem companhia. A escolaridade apresentou uma relação positiva com a prática de corrida e ginástica e negativa com a prática do futebol. Já a renda familiar per capita esteve inversamente associada ao relato de praticar futebol e diretamente associada com a escolha pela atividade de musculação.

\section{Discussão}

Foi constatado em Florianópolis que os homens e os indivíduos mais jovens realizaram atividades físicas mais intensas no lazer. O perfil sociodemográfico dos praticantes das principais atividades físicas de lazer variou conforme o tipo de atividade física principal elegida. A investigação da prática de atividade física no lazer não dispõe, até o presente momento, de uma medida padrão-ouro para sua quantificação. A dificuldade de comparações de resultados com estudos nessa temática fica limitada pela diversidade de procedimentos metodológicos adotados, como diferentes tipos de formas de mensuração, de instrumentos e de pontos de corte utilizados para classificar os sujeitos como ativos nesse domínio. Entretanto, a consistência das evidências permite constatar que, no lazer, os homens são mais ativos do que as mulheres, tanto em termos de prática regular de atividades físicas no tempo livre ${ }^{12,13}$, quanto em termos de gasto energético ${ }^{14}$ e alcance da recomendações de atividade física ${ }^{15}$, quando vinculadas exclusivamente a esse domínio.

O relato da caminhada como principal atividade física realizada no lazer vai ao encontro aos achados da literatura nacional ${ }^{16,17}$ e internacional $^{18}$. A caminhada consiste em uma atividade física simples e acessível à maioria das pessoas. Proporciona inúmeros benefícios à saúde e, por esse motivo, ocupa papel de destaque dentre os exemplos de atividade física voltados ao alcance das recomendações populacionais relacionadas à saúde dos adultos ${ }^{2,19}$. Dentre as atividades esportivas, o futebol foi a opção mais citada. Esse resultado reforça a influência cultural para a hegemonia desse esporte, especialmente entre os homens ${ }^{20}$. Destacou-se ainda o total de praticantes de musculação, que a coloca como a segunda atividade física mais realizada no lazer pela população adulta de Florianópolis. Esses valores foram superiores àqueles observados em outros estudos nacionais ${ }^{20,21}$. A grande preocupação com a estética e a imagem corporal, motivada pela maior exposição do corpo em uma cidade litorânea podem ser responsáveis por tal achado. Já o percentual de praticantes de ciclismo foi muito inferior ao observado em outras localidades brasileiras ${ }^{20,21}$. Questões ambientais como a geografia de Florianópolis, formada por uma grande quantidade de morros e montanhas, e a falta de segurança e mobilidade urbana, podem estar relacionadas com tal achado.

A caminhada, a musculação e a ginástica foram as atividades físicas mais citadas entre as mulheres, assim como em outras pesquisas nacionais $^{16,20,22}$. Dentre os homens, o futebol foi a principal atividade física realizada no lazer, corroborando com outros estudos brasileiros ${ }^{20,21}$. Apesar de ser uma atividade física praticada majoritariamente pelas mulheres, o elevado percentual de homens adultos praticantes de caminhada também tem sido constatado em outras publicações no Brasil ${ }^{23}$ e no exterior ${ }^{24}$. O aparecimento da musculação como a terceira atividade física mais praticada pelos homens é semelhante ao observado em pesquisa desenvolvida no conjunto das capitais brasileiras ${ }^{16}$. Portanto, a evidência das distintas preferências para a prática de atividade física no lazer, conforme gênero, foi ratificada no presente estudo.

A proporção de homens que praticavam atividades físicas intensas no lazer foi quatro vezes superior àquela observada entre as mulheres. Esses achados são consistentes com estudos prévios realizados na Espanha ${ }^{25}$, nos Estados Unidos ${ }^{26} \mathrm{e}$ nas populações da Irlanda e Irlanda do Norte ${ }^{18}$. Podem estar relacionados a esse achado aspectos sociais e culturais. Quando comparados às mulheres, os homens têm um maior engajamento em atividades físicas competitivas, que demandam maior esforço físico ${ }^{23}$. Além disso, quando se comparam os motivos para um lazer ativo, os homens relatam mais frequentemente a recreação como principal razão, enquanto as mulheres relatam maior preocupação com aspectos relacionados à saúde ${ }^{22}$, que independem da necessidade de atividades vigorosas.

As atividades físicas mais intensas praticadas no lazer também foram mais comuns entre os adultos mais jovens. Entretanto, não foram constatadas diferenças significativas entre as idades quando observadas a duração e a frequência semanal de prática de atividades físicas nesse domínio. Provavelmente, isso explica parcialmente o 
decréscimo do alcance às recomendações de atividade física relacionada à saúde entre os adultos mais velhos quando apenas o apenas o domínio do lazer é considerado ${ }^{25}$. Entretanto, é necessário que as políticas públicas enfatizem que a prática de alguma atividade física no cotidiano é melhor para a saúde do que ser inativo. Não cabe negar os benefícios à saúde gerados por práticas mais intensas. Entretanto, na curva de dose-resposta entre a atividade física e os benefícios à saúde, resultados como a redução de indicadores de morbidade e mortalidade por todas as causas em populações adultas são efetivamente alcançados pelo rompimento da barreira da inatividade ${ }^{27}$.

Os principais tipos de atividades físicas realizadas no lazer apresentaram perfis específicos de praticantes. Na presente análise, a caracterização desses sujeitos centrou-se em aspectos sociodemográficos. Em relação ao gênero, estudos precedentes já apontaram que as mulheres se envolvem mais frequentemente com a prática de caminhada e ginástica, enquanto os homens preferem atividades físicas de intensidade mais elevada, como a corrida e o futebol ${ }^{20,23}$. Com relação à faixa etária, a evidência de menor envolvimento dos adultos mais jovens com a prática da caminhada também foi constatada anteriormente ${ }^{16}$. Ao optarem por esse tipo de atividade física, os adultos mais velhos podem estar mais preocupados com a manutenção da saúde do que com outros objetivos, como a estética ou a recreação ${ }^{22}$. Em contrapartida, essa explicação também seria um dos motivos pelo qual os adultos mais jovens estiveram mais engajados com atividades físicas, como a musculação e o futebol.

Poucos estudos têm investigado a associação da cor da pele com a prática de atividade física no lazer. No geral, as evidências têm apontado os adultos com a cor da pele branca como mais ativos nesse domínio ${ }^{28,29}$. Entretanto, no presente estudo, um tipo específico de atividades física no lazer esteve associado aos adultos com cor da pele negra: o futebol. Esse esporte tem sido culturalmente incentivado em nosso país e pode ser considerado como já massificado. Especialmente entre as classes socioeconômicas menos favorecidas, o futebol é visto como uma possibilidade de ascensão social. E, muito embora a população investigada no presente estudo seja adulta, é importante considerar a possibilidade de que indivíduos ativos durante a infância e adolescência tendam a continuar assim na idade adulta ${ }^{30} \mathrm{e}$ de que as experiências bem sucedidas na adolescência possam estar interligadas aos comportamentos e preferências de atividade física durante a idade adulta. Todavia, novas pesquisas são necessárias para explorar com mais profundidade a relação entre as preferências no lazer e a cor da pele.

Os adultos solteiros costumam ser mais engajados com a prática de atividade física no lazer $^{14,28,29}$. O presente estudo, entretanto, observou uma importante associação da caminhada com indivíduos que viviam com companheiro(a). Esse resultado pode ser parcialmente justificado pela possibilidade de uma prática conjunta, o que pode tornar a atividade física mais prazerosa e agradável para quem vive acompanhado, uma vez que a presente análise foi ajustada para variáveis de confundimento, como a idade.

As associações da escolaridade e da renda foram distintas conforme as diferentes atividades físicas no lazer e podem envolver aspectos individuais, como o nível de conhecimento sobre uma determinada modalidade, os interesses e os objetivos de sua prática, assim como questões econômicas e ambientais, como a acessibilidade a espaços adequados, infraestrutura e a segurança dos espaços públicos. Observou-se associação positiva do nível de escolaridade com a prática de corrida e ginástica e negativa com a caminhada e o futebol. Já a musculação foi mais praticada por adultos de maior renda, possivelmente porque a sua prática requer custeio de espaços privados específicos, como academias ou clubes. Menores níveis de renda estiveram associados à prática do futebol com resultados bastante expressivos, onde a diferença percentual entre sua prática pelos adultos mais ricos e mais pobres foi de aproximadamente 20 pontos percentuais em prol dos últimos. Esse resultado demonstra o quanto à prática desse esporte atinge um status de massificação e aceitação pelas classes mais populares no Brasil.

A investigação dos principais tipos de atividades físicas praticadas no lazer e do perfil sociodemográfico de seus praticantes foram pontos fortes do presente estudo, que se deteve ainda em caracterizar a duração, a frequência e a intensidade dessas práticas. Essas questões têm sido pouco exploradas na literatura científica, especialmente em estudos nacionais, e podem subsidiar o estabelecimento de estratégias de estímulo à prática de atividade física no lazer, direcionadas aos interesses e preferências da população adulta. Porém, o instrumento utilizado neste estudo discriminou apenas a principal atividade física realizada, o que impossibilita a compreensão de todas as atividades físicas realizadas nesse domínio pelos adultos.

Novas pesquisas empregando instrumentos com informações mais detalhadas, como os acelerômetros, são recomendadas para estimativas 
ainda mais precisas das características relacionadas à atividade física. Tendo em vista que, independente do aumento da atividade física no lazer, o volume total de atividade física tenha declinado consideravelmente nos últimos anos, sugere-se que as estratégias de promoção da atividade física considerem os interesses populacionais para que sejam estimuladas e expandidas as possibilidades de sua prática nesse domínio.

\section{Colaboradores}

GF Del Duca foi responsável pela concepção do estudo, pela análise estatística e pela redação da primeira versão do manuscrito. MV Nahas supervisionou a análise estatística e participou da redação da primeira versão do manuscrito. PC Hallal contribuiu para a revisão crítica do texto. KG Peres coordenou o trabalho de campo e contribuiu para a revisão crítica do texto.

\section{Referências}

1. Lee IM, Shiroma EJ, Lobelo F, Puska P, Blair SN, Katzmarzyk PT. Effect of physical inactivity on major non-communicable diseases worldwide: an analysis of burden of disease and life expectancy. The Lancet 2012; 380(9838):219-229.

2. World Health Organization (WHO). Global health risks: mortality and burden of disease attributable to selected major risks. Geneva: WHO; 2009.

3. Ng SW, Norton EC, Popkin BM. Why have physical activity levels declined among Chinese adults? Findings from the 1991-2006 China Health and Nutrition Surveys. Soc Sci Med 2009; 68(7):1305-1314.

4. Juneau CE, Potvin L. Trends in leisure-, transport-, and work-related physical activity in Canada 1994-2005. Prev Med 2010; 51(5):384-386.

5. Borodulin K, Laatikainen T, Juolevi A, Jousilahti P. Thirty-year trends of physical activity in relation to age, calendar time and birth cohort in Finnish adults. Eur J Public Health 2008; 18(3):339-344.

6. Autenrieth CS, Baumert J, Baumeister SE, Fischer B, Peters A, Döring A, Thorand B. Association between domains of physical activity and all-cause, cardiovascular and cancer mortality. Eur J Epidemiol 2011; 26(2):91-99.

7. United States of America. Centers for Disease Control and Prevention. Health risks in the United States: Behavioral Risk Factor Surveillance System. At a glance 2010. Atlanta: Centers for Disease Control and Prevention; 2010.

8. Brasil. Ministério da Saúde (MS). Vigitel Brasil 2009: vigilância de fatores de risco e proteção para doenças crônicas por inquérito telefônico. Brasília: MS; 2010.

9. Gomes VB, Siqueira KS, Sichieri R. Atividade física em uma amostra probabilística da população do $\mathrm{Mu}$ nicípio do Rio de Janeiro. Cad Saude Publica 2001; 17(4):969-976.

10. Programa das Nações Unidas para o Desenvolvimento [PNUD]. Atlas do Desenvolvimento Humano no Brasil Ranking Municipal 2010. 2013. [acessado 2013 jul 31]. Disponível em: http://www.atlasbrasil.org.br/2013/ ranking. 
11. Instituto Brasileiro de Geografia e Estatística (IBGE). Manual do Recenseador - Censo 2000. Rio de Janeiro: IBGE; 2000.

12. Martinez-Gonzalez MA, Varo JJ, Santos JL, De Irala J, Gibney M, Kearney J, Martínez JA. Prevalence of physical activity during leisure time in the European Union. Med Sci Sports Exerc 2001; 33(7):1142-1146.

13. Knuth AG, Malta DC, Dumith SC, Pereira CA, Neto OLM, Temporão JG, Penna G, Hallal PC. Prática de atividade física e sedentarismo em brasileiros: resultados da Pesquisa Nacional por Amostra de Domicílios (PNAD)-2008. Cien Saude Colet 2011; 16(9):36973705.

14. Meseguer CM, Galan I, Herruzo R, Zorrilla B, Rodriguez-Artalejo F. Leisure-time physical activity in a southern European mediterranean country: adherence to recommendations and determining factors. Rev Esp Cardiol 2009; 62(10):1125-1133.

15. Malta DC, Silva SA, Oliveira PPV, Iser BPM, Bernal RTI, Sardinha LMV, Moura L. Monitoring of risk and protective factors for chronic non communicable diseases by telephone survey in Brazilian State Capitals, 2008. Rev Bras Epidemiol 2012; 15(3):639-650.

16. Malta DC, Moura EC, Castro AM, Cruz DKA, Morais Neto OL, Monteiro CA. Padrão de atividade física em adultos brasileiros: resultados de um inquérito por entrevistas telefônicas, 2006. Epidemiologia e Serviços de Saúde 2009; 18(1):7-16.

17. Sá Silva SPd, Sandre-Pereira G, Salles-Costa R. Fatores sociodemográficos e atividade física de lazer entre homens e mulheres de Duque de Caxias/RJ; Socio-demographic factors and leisure-time physical activity among men and women of Duque de Caxias/RJ. Cien Saude Colet 2011; 16(11):4491-4501.

18. Livingstone M, Robson P, McCarthy S, Kiely M, Harrington K, Browne P, Galvin M, Wareham NJ, Rennie KL. Physical activity patterns in a nationally representative sample of adults in Ireland. Public Health Nutr 2001; 4(5A):1107-1116.

19. United States. Department of Health and Human Services. Physical Activity Guidelines for Americans: be acti$v e$, healthy, and happy! Washington: Secretary of Health and Human Services; 2008.

20. Dumith SC, Domingues MR, Gigante D. Epidemiologia das atividades físicas praticadas no tempo de lazer por adultos do Sul do Brasil. Rev Bras Epidemiol 2009; 12(4):646-658.
21. Zanchetta LM, Barros MB, Cesar CL, Carandina L, Goldbaum M, Alves MC. Inatividade física e fatores associados, São Paulo, Brasil. Rev Bras Epidemiol 2010; 13(3):387-399.

22. Monteiro CA, Conde WL, Matsudo SM, Matsudo VR, Bonseñor IM, Lotufo PA. A descriptive epidemiology of leisure-time physical activity in Brazil, 1996-1997. Rev Panam Salud Publica 2003; 14(4):246-254.

23. Salles-Costa R, Heilborn ML, Werneck GL, Faerstein E, Lopes CS. Gênero e prática de atividade física de lazer. Cad Saude Publica 2003; 19(Supl. 2):S325-S33.

24. de Munter JSL, Charles A, Anton K, Karien S. Large ethnic variations in recommended physical activity according to activity domains in amsterdam, the netherlands. Int J Behav Nutr Phys Act 2010; 7:85.

25. Meseguer CM, Galan I, Herruzo R, Rodriguez-Artalejo F. Trends in leisure time and occupational physical activity in the Madrid region, 1995-2008. Rev Esp Cardiol 2011; 64(1):21-27.

26. He XZ, Baker DW. Differences in leisure-time, household, and work-related physical activity by race, ethnicity, and education. J Gen Intern Med 2005; 20(3): 259-266.

27. Löllgen H, Böckenhoff A, Knapp G. Physical activity and all-cause mortality: an updated meta-analysis with different intensity categories. Int J Sports Med 2009; 30(3):213-224.

28. Marshall SJ, Jones DA, Ainsworth BE, Reis JP, Levy SS, Macera CA. Race/ethnicity, social class, and leisure-time physical inactivity. Med Sci Sports Exerc 2007; 39(1):44-51.

29. Del Duca GF, Nahas MV, Garcia LMT, Mota J, Hallal PC, Peres MA. Prevalence and sociodemographic correlates of all domains of physical activity in Brazilian adults. Preventive Medicine 2013; 56(2):99-102.

30. Azevedo MR, Araújo CL, Silva MC, Hallal PC. Tracking of physical activity from adolescence to adulthood: a population-based study. Rev Saude Publica 2007; 41(1):69-75.

Artigo apresentado em 11/09/2013

Aprovado em 05/10/2013

Versão final apresentada em 09/10/2013 